\title{
Recognizing the involvement of early-career researchers in peer review
}

\author{
The Nature Reviews journals have started an initiative to encourage the involvement of \\ early-career researchers in our peer review process, to aid with career development and ensure \\ proper acknowledgement. Nature Reviews Endocrinology is proud to support this scheme.
}

If Nature

Reviews

Endocrinology

has committed

to ... ensuring

that everyone

involved in our

peer review

process is

appropriately

recognized $y$
Nature Reviews Endocrinology is committed to supporting the development of the next generation of researchers. Early-career researchers (ECRs) drive science forward with new ideas and enthusiasm, but a lack of opportunities for training in key skill areas, such as peer reviewing manuscripts, can hinder career development. In addition, the efforts of ECRs, which are many and varied, are sometimes not acknowledged correctly or at all. For example, in one survey of ECRs in the life sciences, $92 \%$ of respondents reported that they had been involved in the peer review process, and more than half did so with no involvement of their supervisor ${ }^{1,2}$. To address these issues, Nature Reviews Endocrinology has committed to facilitating training in peer review and to ensuring that everyone involved in our peer review process is appropriately recognized (https://www.nature. $\mathrm{com} /$ nrendo/for-referees/reviewer-initiatives).

The peer review process is integral to ensuring the integrity of the scientific literature. Therefore, the ability to peer review manuscripts is an essential skill for any researcher. Not only does peer review enable critical appraisal of the latest advancements in the field with a view to ensuring integrity of scientific research, but it also supports clear and effective communication. With these points in mind, the ECR peer review initiative will help ensure tomorrow's established researchers develop the key skills needed to help maintain the validity of the scientific record.

We are aware that ECRs require training and support to aid career development. The Nature Reviews ECR peer review initiative, which launched in March 2020, adds to Nature Research's portfolio of training resources on peer review (https://masterclasses.nature. com/focus-on-peer-review-online-course/16605550) and takes further steps to address the training needs of ECRs. The initiative aims:

- To ensure ECRs receive proper credit for their contribution to the peer review process.

- To encourage training of ECRs on how to peer review Review articles.
- To expand the pool of well-trained reviewers.

- To help ensure the next generation of established scientists recognize peer review as a scholarly contribution.

In this initiative, we offer invited referees the option to include an ECR, such as a PhD student or postdoc, as a co-referee. By doing so, referees can mentor ECRs throughout the peer review process, thereby facilitating key training in scholarly communication, critical assessment and providing collegial feedback. This training is especially useful when assessing Review articles, which have different requirements to the often more familiar evaluation of primary research papers. The peer review report will be considered a co-review, meaning that both the ECR and supervisor receive recognition for their contributions in our journal records; each referee's activity, but not the peer review reports, will also be visible to other journals in the Nature family. This recognition extends to being formally acknowledged by name in our published articles, which is an option for all our referees. In addition, ECRs and supervisors will also be able to register their reviewing activity for Nature Reviews Endocrinology and other participating journals on their ORCID profile. Thus far, we have received a very positive reaction from the community. Since the scheme launched, $41 \%$ of manuscripts sent out for review have had at least one ECR involved. Feedback from those involved has been positive and the hope is that more journals will offer similar schemes in the future.

We feel strongly that ECRs deserve opportunities to receive training in the skills they require for career development. We are proud to be able to have a role in this training process and we hope that our readers will join us in supporting this initiative.

1. McDowell, G. S. et al. Co-reviewing and ghostwriting by early career researchers in the peer review of manuscripts. eLife $\mathbf{8}$, e48425 (2019).

2. Inside eLife. Early-career researchers: Views on peer review. eLife https://elifesciences.org/inside-elife/982053f4/early-careerresearchers-views-on-peer-review (2018). 